\title{
Delivery of curcumin by directed self-assembled micelles enhances therapeutic treatment of non-small-cell lung cancer
}

This article was published in the following Dove Press journal:

International Journal of Nanomedicine

3 April 2017

Number of times this article has been viewed

\author{
Wen-Ting Zhu ${ }^{1,2, *}$ \\ Sheng-Yao Liü,* \\ Lei $\mathrm{Wu}^{1,2}$ \\ Hua-Li Xu ${ }^{4}$ \\ Jun Wang ${ }^{1,2}$ \\ Guo-Xin $\mathrm{Ni}^{3,5}$ \\ Qing-Bing Zeng ${ }^{1,2}$
}

'Biomaterial Research Center, School of Pharmaceutical Sciences, Southern Medical University, Guangzhou, China; ${ }^{2}$ Guangdong Provincial Key Laboratory of New Drug Screening, School of Pharmaceutical Sciences, Southern Medical University, Guangzhou, China; ${ }^{3}$ Department of Orthopeadics and Traumatology, Nanfang Hospital, Southern Medical University, Guangzhou, China; ${ }^{4}$ Department of Anesthesiology, Zhujiang Hospital, Southern Medical University, Guangzhou, China; ${ }^{5}$ Department of Rehabilitation Medicine, First Affiliated Hospital, Fujian Medical University, Fuzhou, China

*These authors contributed equally to this work

Correspondence: Qing-Bing Zeng Biomaterial Research Center, School of Pharmaceutical Sciences, Southern Medical University, 1023 Southern Shatai Street, Guangzhou, GD 510515, China $\mathrm{Tel} / \mathrm{fax}+862062789462$

Email zengqb@smu.edu.cn

Guo-Xin Ni

Department of Orthopaedics and Traumatology, Nanfang Hospital, Southern Medical University, 1838 Guangzhou Avenue (N), Guangzhou, GD 5105I5, China

Tel/fax +86206164 I744

Email guoxinnil5tg@I63.com
Background: It has been widely reported that curcumin (CUR) exhibits anticancer activity and triggers the apoptosis of human A549 non-small-cell lung cancer (NSCLC) cells. However, its application is limited owing to its poor solubility and bioavailability. Therefore, there is an urgent need to develop a new CUR formulation with higher water solubility and better biocompatibility for clinical application in the future.

Materials and methods: In this study, CUR-loaded methoxy polyethylene glycol-polylactide (CUR/mPEG-PLA) polymeric micelles were prepared by a thin-film hydration method. Their characteristics and antitumor effects were evaluated subsequently.

Results: The average size of CUR/mPEG-PLA micelles was $34.9 \pm 2.1 \mathrm{~nm}$ with its polydispersity index (PDI) in the range of $0.067-0.168$. The encapsulation efficiency and drug loading were $90.2 \% \pm 0.78 \%$ and $9.1 \% \pm 0.07 \%$, respectively. CUR was constantly released from the CUR/ mPEG-PLA micelles, and its cellular uptake in A549 cells was significantly increased. It was also found that CUR/mPEG-PLA micelles inhibited A549 cell proliferation, increased the cell cytotoxicity, induced G2/M stage arrest and promoted cell apoptosis. Moreover, the CUR/mPEGPLA micelles suppressed the migration and invasion of A549 cells more obviously than free CUR. Additionally, CUR/mPEG-PLA micelles inhibited human umbilical vein endothelial cells migration, invasion and corresponding tube formation, implying the antiangiogenesis ability. Its enhanced antitumor mechanism may be related to the reduced expression of vascular endothelial growth factor, matrix metalloproteinase (MMP)-2, MMP-9 and Bcl-2 as well as the increased expression of Bax.

Conclusion: The mPEG-PLA copolymer micelles can serve as an efficient carrier for CUR. The CUR/mPEG-PLA micelles have promising clinical potential in treating NSCLC.

Keywords: curcumin, mPEG-PLA, polymeric micelles, A549 cells, HUVECs, angiogenesis

\section{Introduction}

Lung cancer is one of the malignant tumors with the highest morbidity and mortality in the world, among which non-small-cell lung cancer (NSCLC) accounts for 85\% of cases. ${ }^{1}$ Chemotherapy is the main strategy for those with advanced lung cancer or unsuitable for surgery with metastasis. ${ }^{2}$ Unfortunately, the use of traditional chemotherapy drug is limited by its unsatisfactory efficacy, poor selectivity, high drug resistance and toxicity to normal tissue. ${ }^{3}$ Therefore, it is necessary to develop novel drug formulations with high efficacy but low toxicity.

Curcumin (CUR), a natural polyphenolic compound isolated from the rhizome of Curcuma longa, has the potential to inhibit cell survival, proliferation, invasion, migration and angiogenesis. ${ }^{4-6}$ A variety of signaling pathways, including Cyclin D1, 
COX-2, nuclear factor-kappa B (NF- $\mathrm{B}$ ) and matrix metalloproteinases (MMPs), have been shown to be involved in the multi-therapeutic activities of CUR. ${ }^{7,8}$ More importantly, no sign of toxicity was observed in animals or humans treated with CUR, indicating its in vivo safety. ${ }^{9}$ However, its clinical application is still restricted by poor solubility in aqueous solution, poor oral bioavailability and extensive first-pass metabolism. ${ }^{10}$

Nanotechnology has been widely used in the field of drug delivery and cancer therapy due to its advantages in developing aqueous-based formulations for hydrophobic drugs. ${ }^{11-13}$ For example, liposomes, nanotubes and polymer nanoparticles have been successfully used as drug delivery systems (DDSs) to develop aqueous formulations and improve antitumor efficacy. ${ }^{14-16}$ Recently, scientists have shown interest in amphiphilic polymer micelles owing to their core-shell geometry ${ }^{17,18}$ and excellent characteristics, such as low toxicity, good biocompatibility and high biodegradability. ${ }^{19}$ As a perfect example, methoxy polyethylene glycol-polylactide (mPEG-PLA) polymeric micelles are composed of hydrophilic mPEG and hydrophobic PLA. On one hand, mPEG can act as a hydrophilic shell to reduce the nonspecific uptake by the reticuloendothelial system (RES), thus prolonging the circulation time in blood. ${ }^{20}$ PLA, on the other hand, mainly acts as hydrophobic core for drug loading (DL). More recently, mPEG-PLA polymeric micelles have been proven as excellent DDSs for CUR. ${ }^{21}$ However, the effect of CUR-loaded mPEG-PLA polymeric micelles (CUR/mPEG-PLA micelles) for treating NSCLC remains unknown.

Hence, CUR/mPEG-PLA micelles were prepared in this study. Subsequently, their efficiency was examined at the cellular level by investigating the drug uptake, apoptosis and cytotoxicity to A549 cells in vitro. More importantly, the anti-angiogenesis effect on human umbilical vein endothelial cells (HUVECs) was evaluated.

\section{Materials and methods}

\section{Materials and cell lines}

3-(4,5-Dimethylthiazol-2-yl)-2,5-di-phenyltetrazolium bromide (MTT) and Hoechst 33258 were purchased from Sigma (St Louis, MO, USA). CUR was obtained from Aladdin (Shanghai, China) and Matrigel from BD Biosciences (San Jose, CA, USA). Anti-Bcl-2, anti-Bax, anti-MMP-2 and anti-MMP-9 were provided by Abcam (Cambridge, UK), while anti-vascular endothelial growth factor (VEGF) was obtained from Boster (Wuhan, China). The secondary antibody goat anti-rabbit-IgG horseradish peroxidase (HRP) was from Jackson ImmunoResearch Laboratories Inc (West Grove, PA, USA).

mPEG (molecular weight $=5000 \mathrm{Da}$ ) and stannous 2-ethylhexanoate were obtained from Aladdin. D,L-Lactide was obtained from Dibo (Shanghai, China). The mPEGPLA diblock copolymers were synthesized by ring-opening polymerization reaction following a previously reported procedure. ${ }^{22}$ Briefly, $2.5 \mathrm{~g}$ of pure D,L-lactide and $5 \mathrm{~g}$ of mPEG were placed in a dried round-bottomed flask. The reactants were gently heated with stirring till they turned into liquid. Stannous octoate was added as a catalyst into the reaction mixture. The flask was sealed under nitrogen, and the copolymerization was carried out at $130^{\circ} \mathrm{C}$ for 24 hours. The crude product was dissolved in dichloromethane and then purified by precipitation in ice cold diethyl ether followed by filtration. The obtained $\mathrm{MPEG}-\mathrm{PLA}$ copolymer was characterized by Fourier transform infrared spectroscopy (FTIR, Nicolet 6700; Thermo Fisher, Grand Island, $\mathrm{NY}, \mathrm{USA})$ and ${ }^{1} \mathrm{H}$ nuclear magnetic resonance ( $\left.{ }^{1} \mathrm{H}-\mathrm{NMR}\right)$ spectroscopy (AVANCE III $400 \mathrm{MHz}$; Bruker, Bern, Switzerland). The number average molecular weight of mPEG-PLA copolymer was 7,402 Da, calculated by ${ }^{1} \mathrm{H}$ NMR (data not shown).

The human lung cancer A549 cells and HUVECs were purchased from the Type Culture Collection of the Chinese Academy of Sciences (Shanghai, China). They were cultured in Roswell Park Memorial Institute (RPMI)-1640 (Gibco, Grand Island, NY, USA) supplemented with fetal bovine serum (FBS; Gibco). HUVECs were cultured in Dulbecco's Modified Eagle's Medium (DMEM) containing 10\% FBS. All the abovementioned cells were maintained at $37^{\circ} \mathrm{C}$ in a humidified incubator containing $5 \% \mathrm{CO}_{2}$. Permission for this study was obtained from the Ethics Committee of Southern Medical University.

\section{Preparation and characterization of CUR/mPEG-PLA micelles \\ Encapsulation of CUR in mPEG-PLA micelles}

CUR/mPEG-PLA micelles were prepared by thin-film hydration method. Briefly, $10 \mathrm{mg}$ of CUR and $90 \mathrm{mg}$ of mPEG-PLA copolymer were co-dissolved in $10 \mathrm{~mL}$ of methanol under mild stirring. Then, the solution was evaporated in rotary evaporator at $60^{\circ} \mathrm{C}$. During this process, homogenous co-evaporation was obtained, and CUR was distributed in mPEG-PLA copolymer as an amorphous substance. Subsequently, the co-evaporation was dissolved in water at $60^{\circ} \mathrm{C}$ to self-assemble into micelles with CUR encapsulated inside. The CUR/mPEG-PLA micelles solution 
was filtered through a $0.22 \mu \mathrm{m}$ syringe filter(Jinteng, Tianjin, China), lyophilized and stored at $4{ }^{\circ} \mathrm{C}$ prior to use.

\section{Particle size and zeta potential}

The prepared blank mPEG-PLA micelles and CUR/mPEGPLA micelles were assessed by Malvern-3000Hs particle size analyzer at $25^{\circ} \mathrm{C}$ for parameters such as particle size distribution, polydispersity index (PDI) and zeta potential. For each sample, such assessment was taken three times, and the average results were obtained.

\section{Morphology of copolymer micelles}

The morphological characteristics of CUR/mPEG-PLA micelles were examined by transmission electron microscope (TEM; H-7650; Hitachi, Tokyo, Japan). The CUR/mPEGPLA micelles were diluted with distilled water and placed on a copper grid covered with nitrocellulose. Samples were negatively stained with $2 \%$ phosphotungstic acid and dried at room temperature.

\section{X-ray diffraction (XRD)}

Crystallographic assays were performed on CUR powder, a physical mixture of CUR and MPEG-PLA, lyophilized blank mPEG-PLA micelles and lyophilized CUR/mPEGPLA micelles powder using an X-ray diffractometer (D8 ADVANCE; Bruker, Ettlingen, Germany). The samples were scanned from $5^{\circ}$ to $50^{\circ}$ at a scanning speed of $1 / \mathrm{min}$ and a step size of $0.02^{\circ}$. The X-ray tube was operated at a potential of $40 \mathrm{kV}$ and a current of $40 \mathrm{~mA}$.

\section{Differential scanning calorimetry (DSC)}

The physical status of CUR powder, lyophilized blank mPEG-PLA micelles and lyophilized CUR/mPEG-PLA micelles powder was studied by Simultaneous Thermal Analyzer (STA449 F3; NETZSCH, Ahlden, Germany) under nitrogen atmosphere at a flow rate of $20 \mathrm{~mL} / \mathrm{min}$. The temperature gradually increased from $20^{\circ} \mathrm{C}$ to $250^{\circ} \mathrm{C}$ with a changing rate of $10^{\circ} \mathrm{C} / \mathrm{min}$.

\section{Entrapment efficiency (EE) and DL}

The concentration of CUR was determined by highperformance liquid chromatography (HPLC; LC-20A; Shimadzu, Tokyo, Japan) with sample being diluted before measurement. Detection was taken on a Shimadzu SPDM20A detector. Chromatographic separations were performed on a Diamonsil ODS C18 column $(250 \times 4.6 \mathrm{~mm}$, pore size $5 \mu \mathrm{m}$; Dikma, Beijing, China). The column temperature was kept at $28^{\circ} \mathrm{C}$. Acetonitrile $/ 2.5 \%$ acetic acid $(58 / 42, \mathrm{v} / \mathrm{v})$ was used as eluent at a flow rate of $1 \mathrm{~mL} / \mathrm{min}$. EE and DL of CUR/mPEG-PLA micelles were determined as follows. Briefly, $10 \mathrm{mg}$ of lyophilized CUR/mPEG-PLA micelles were dissolved in $0.1 \mathrm{~mL}$ of methanol to disrupt and release CUR, and then, the solution was centrifuged at $12,000 \mathrm{rpm}$ for $5 \mathrm{~min}$. The supernatant was determined by HPLC. The $\mathrm{EE}$ and DL were expressed by the equation:

$$
\begin{gathered}
\mathrm{EE}=\frac{\text { Actual drug loading }}{\text { Theoretical drug loading }} \times 100 \% \\
\mathrm{DL}=\frac{\text { Drug }}{\text { Polymer }+ \text { Drug }} \times 100 \%
\end{gathered}
$$

\section{In vitro drug release}

In vitro drug release behaviors of CUR/mPEG-PLA micelles and free CUR were evaluated by the dialysis membrane method. Briefly, $1 \mathrm{~mL}$ solution (either CUR/mPEG-PLA micelles or free CUR solution) was placed in dialysis bags (molecular mass cutoff is $3.5 \mathrm{kDa}$ ). The dialysis bags were incubated in $40 \mathrm{~mL}$ of phosphate-buffered saline (PBS) ( $\mathrm{pH}=7.4)$ containing Tween $80(0.5 \mathrm{wt} \%)$ at $37^{\circ} \mathrm{C}$ with gentle shaking. At predetermined time point, release medium samples were withdrawn and replaced with fresh release medium. The samples were centrifuged, and then, the supernatant was collected and stored at $-20^{\circ} \mathrm{C}$ till analysis. The released CUR samples were quantified by HPLC.

\section{Cellular uptake of CUR/mPEG-PLA micelles}

Cellular uptake of free CUR and CUR/mPEG-PLA micelles was measured by confocal microscopy (FV10i-w; Olympus, Tokyo, Japan) and flow cytometry (FCM; BD Biosciences) analysis, respectively. A549 cells were grown on a confocal dish in complete medium. After 24 hours, the medium were removed, and cells were treated with serumfree medium containing either free CUR $(20 \mu \mathrm{g} / \mathrm{mL})$, CUR/ mPEG-PLA micelles $(20 \mu \mathrm{g} / \mathrm{mL})$ or blank mPEG-PLA micelles. After incubation for 0,1 and 4 hours, the medium were removed and washed with PBS. Subsequently, cells were stained with Hoechst 33258 for 5 minutes, subsequently washed with PBS and fixed with cold $4 \%$ paraformaldehyde for 10 minutes. Thereafter, they were washed again with PBS and examined using a confocal microscopy.

For FCM analysis, cells were collected and washed with PBS. Then, intracellular CUR fluorescence was analyzed by FCM after excitation with a $488 \mathrm{~nm}$ argon laser. Fluorescence emission at $520-530 \mathrm{~nm}$ from 10,000 cells was collected, amplified and scaled to generate single parameter histogram. 


\section{Cytotoxicity study}

The cytotoxicity assay of free CUR or CUR/mPEG-PLA micelles was evaluated by MTT method. Cells were plated at a density of $1 \times 10^{4}$ cells per well in 96-well plates and grown for 24 hours. Then, cells were exposed to a series of free CUR, CUR/mPEG-PLA micelles or blank mPEG-PLA micelles at different concentrations for 48 hours, respectively. After the incubation period, the cells were treated with $5 \mathrm{mg} / \mathrm{mL}$ of MTT solution for 4 hours, then the solution was removed and $150 \mu \mathrm{L}$ of dimethyl sulfoxide (DMSO) was added into each well. Absorbance intensity was measured by using a microplate reader (iMark; Bio-Rad, Hercules, CA, USA) at $570 \mathrm{~nm}$.

\section{Colony formation unit assay}

About $1 \times 10^{3}$ cells were added into each well of a six-well culture plate (three wells for each group). After incubation at $37^{\circ} \mathrm{C}$ for 14 days, the cells were washed twice with PBS and stained with $0.1 \%$ crystal violet solution. The number of colonies containing $\geq 20$ cells was counted under a microscope.

\section{Apoptosis assay and cell cycle analysis}

FCM assay was performed to investigate the cell cycle arrest and apoptotic effect of CUR/mPEG-PLA micelles. A549 cells cultured in six-well plates were treated with blank mPEG-PLA micelles, free CUR $(20 \mu \mathrm{g} / \mathrm{mL})$ and CUR/ mPEG-PLA micelles $(20 \mu \mathrm{g} / \mathrm{mL})$ for 24 hours, and medium without treatment was considered as control. For the cell cycle analysis, they were washed with PBS, fixed with $70 \%$ ethanol overnight, pretreated with $100 \mu \mathrm{L}$ RNase A and stained with $400 \mu \mathrm{L}$ propidium iodide (PI) for 30 minutes. Cell apoptosis was determined using the Annexin V-fluorescein isothiocyanate (FITC)/PI detection kit (KeyGEN BioTECH, Nanjing, China). The cells were collected and then washed twice with PBS, gently resuspended in $500 \mu \mathrm{L}$ of binding buffer and stained with $5 \mu \mathrm{L}$ of Annexin V-FITC and $5 \mu \mathrm{L}$ of PI solution according to the manufacturer's instructions.

\section{Caspase 3 activity}

The activity of caspase 3 was measured using caspase 3 activity assay kits (Nanjing Jiancheng Bioengineering Institute, Nanjing, China) according to the manufacturer's protocol. The assay is based on spectrophotometric detection of the chromatophore $\rho$-nitroaniline $(\rho \mathrm{NA}$ ) after cleavage from the labeled substrate Ac-DEVD- $\rho$ NA by caspase 3 protease. The level of caspase 3 activity was tested at $405 \mathrm{~nm}$ in a microplate reader. Fold increase in caspase 3 activity was determined by comparing the absorbance from an apoptotic sample with an untreated control after subtracting the background value reading from cell lysates and buffers.

\section{Tube formation assay of HUVECs}

The tube formation assay was performed as described previously. ${ }^{23} \mathrm{~A} 24$-well plate was coated with $100 \mu$ L Matrigel per well and allowed to polymerize for 2 hours. Then, HUVECs suspended in DMEM medium were seeded on to the Matrigel, and the cells were treated with either blank mPEG-PLA micelles, free CUR or CUR/mPEG-PLA micelles. After 6 hours, images were taken by a digital camera (IX83; Olympus, Toyko, Japan) attached to an inverted microscope.

\section{Wound healing and transwell invasion}

For migration assay, wound-healing assay was done. HUVECs and A549 cells were seeded in a six-well dish and incubated for 24 hours; monolayer was then scratched with pipette tips and washed with PBS. Subsequently, fresh medium containing either blank mPEG-PLA micelles, free CUR or CUR/ mPEG-PLA micelles was added to the scratched HUVECs and A549 cells. Medium without treatment was considered as control. Photographs were taken at 0 and 24 hours in an inverted microscope. The migrated cells were quantified by image-pro-plus (IPP) software, and the percentage of inhibition was calculated on the basis of untreated cells as 100 .

For invasion assays, $5 \times 10^{4}$ cells in serum-free medium were placed in the upper chamber of the transwell plate (24-well insert, pore size: $8 \mu \mathrm{m}$ ) coated with Matrigel, and the lower chambers were filled with $10 \%$ FBS medium. The blank mPEG-PLA micelles, free CUR and CUR/mPEGPLA micelles were added to the upper chamber, respectively. After 24 hours of incubation at $37^{\circ} \mathrm{C}$, noninvasive cells were scraped, and migrated cells were fixed with $4 \%$ paraformaldehyde and stained with $0.05 \%$ crystal violet. The invasive cells were quantified by IPP software, and the percentage of invasive cells inhibited by CUR/mPEG-PLA micelles was calculated using untreated cells as 100 .

\section{Western blot}

The total cell proteins were extracted using Radio Immunoprecipitation Assay (RIPA) lysis buffer containing 1\% phenylmethylsulfonyl fluoride (PMSF) and 1\% protease inhibitors and then separated by sodium dodecyl sulfatepolyacrylamide gel electrophoresis (SDS-PAGE). The proteins were transferred on to nitrocellulose filter membranes. The membranes were blocked in 5\% fat-free dry milk for 2 hours and incubated with specific primary antibody in an appropriate dilution ratio including Bax $(1: 2,500)$, BcL-2 
(1:1,000), VEGF (1:200), MMP-2 (1:2,500), MMP-9 $(1: 5,500)$ and glyceraldehyde 3 -phosphate dehydrogenase (GAPDH; 1:1,000) overnight at $4{ }^{\circ} \mathrm{C}$ and incubated with the corresponding secondary antibodies. Specific protein bands were visualized using an enhanced chemiluminescence (ECL) advanced Western blot detection kit (Merck Millipore, Billerica, MA,USA).

\section{Statistical analysis}

The results were expressed as the mean \pm standard deviation (SD). Statistical analysis was performed with one-way analysis of variance (ANOVA) using the SPSS 13.0 software. $P<0.05$ denoted significance in all cases.

\section{Results}

\section{Preparation and characterization of CUR/mPEG-PLA micelles}

Figure 1A illustrates CUR and mPEG-PLA self-assembled into CUR/mPEG-PLA micelles, which encapsulated CUR in the core part, with a hydrophilic shell. As shown in Figure 1B, the average particle size, PDI and zeta potential of obtained

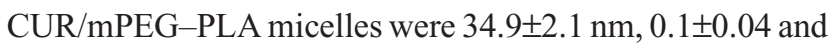
$0.90 \pm 0.35 \mathrm{mV}$, respectively. Furthermore, TEM imaging revealed that CUR/mPEG-PLA micelles were spherical in aqueous solution and monodisperse with a mean diameter of $\sim 24 \mathrm{~nm}$ (Figure 1C). HPLC assay was used to determine CUR concentrations in the micelles. The EE and DL of these CUR/mPEG-PLA micelles were 90.2\% $\pm 0.78 \%$ and $9.1 \% \pm 0.07 \%$, respectively.

The in vitro release of CUR from micelles was investigated by dialysis under sink conditions with a $0.5 \%$ Tween 80 solution in PBS (pH 7.4) as the release medium. As shown in Figure 1D, $90.6 \% \pm 2.2 \%$ of free CUR was released into the medium in the first 48 hours, whereas only $34.9 \% \pm 1.9 \%$ of CUR from the CUR/mPEG-PLA micelles. The cumulative release rate from CUR/mPEG-PLA micelles was $52.4 \% \pm 2.8 \%$ over 5 days, compared with $94.6 \% \pm 1.6 \%$ from free CUR.

The physical form of the drug encapsulated in the polymeric matrix was evaluated by XRD and DSC analysis. The $\mathrm{XRD}$ spectra are presented in Figure 2A, in comparison with
A

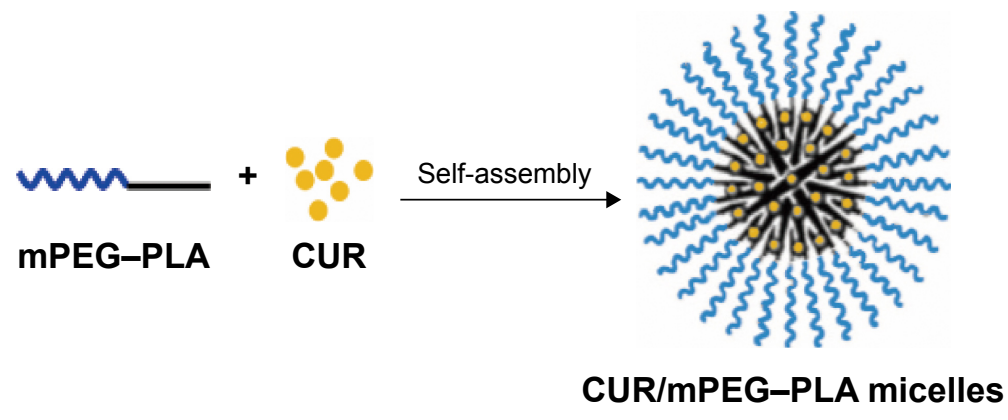

CUR/mPEG-PLA micelles
B Size distribution(s)

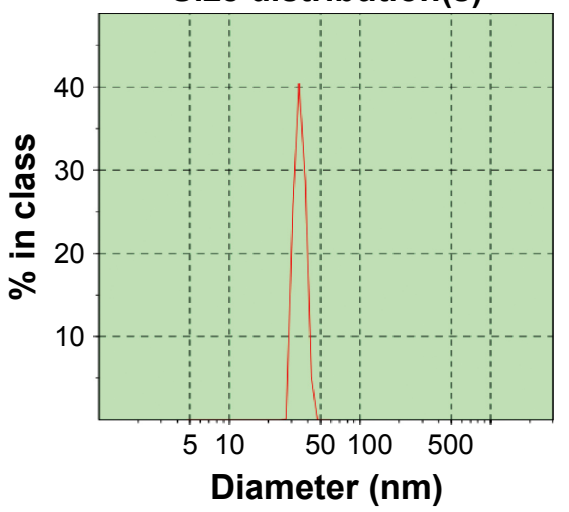

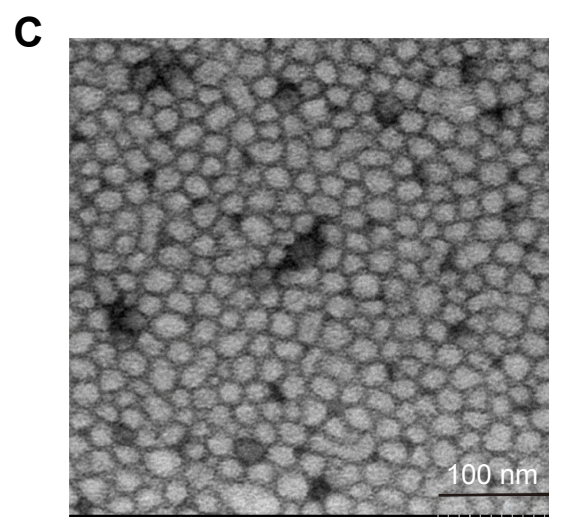

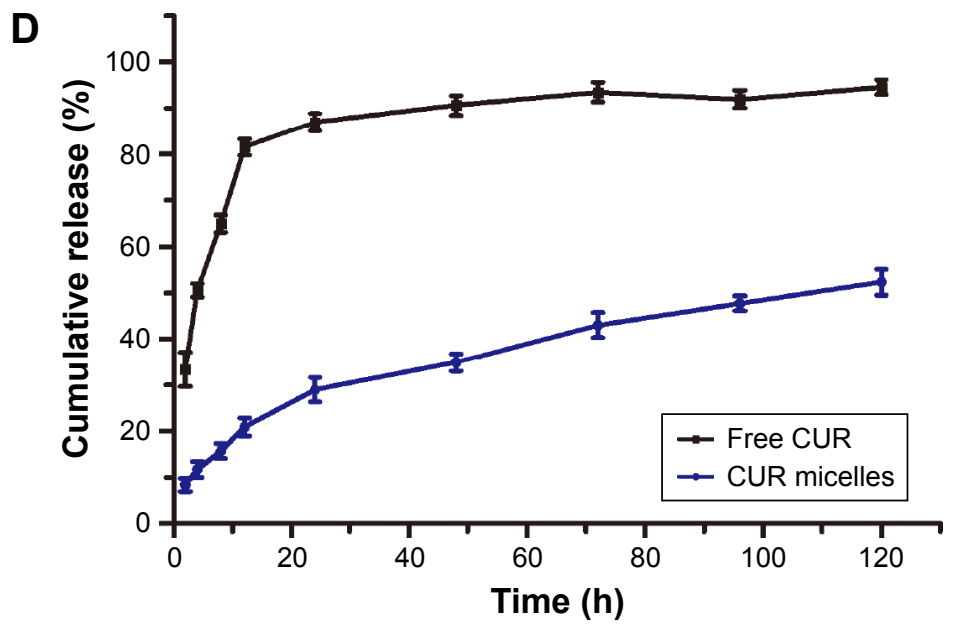

Figure I Preparation and characterization of CUR micelles.

Notes: (A) Preparation scheme of CUR micelles. (B) Particle size distribution of CUR micelles. (C) TEM image of CUR micelles. (D) In vitro drug release profiles of free CUR and CUR micelles in PBS (pH 7.4). CUR micelles, CUR/mPEG-PLA micelles.

Abbreviations: CUR, curcumin; mPEG-PLA, methoxy polyethylene glycol-polylactide; PBS, phosphate-buffered saline; TEM, transmission electron microscope. 
A

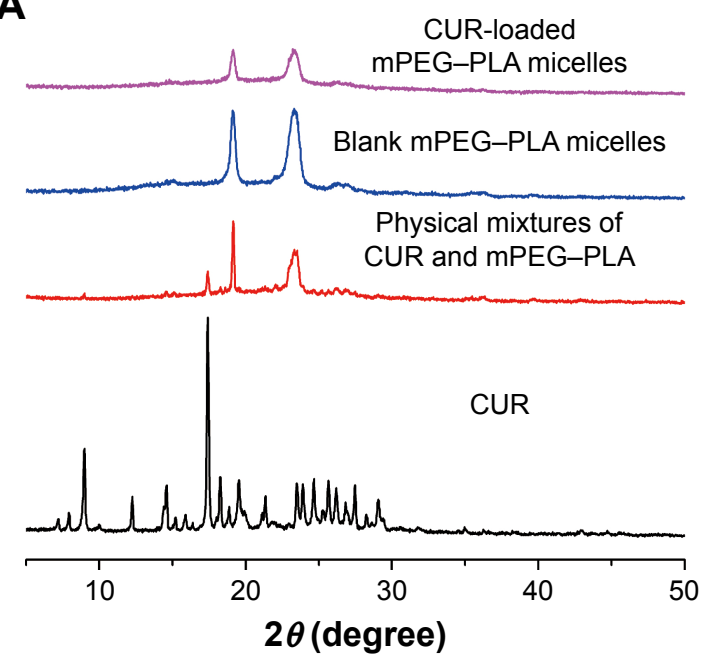

B

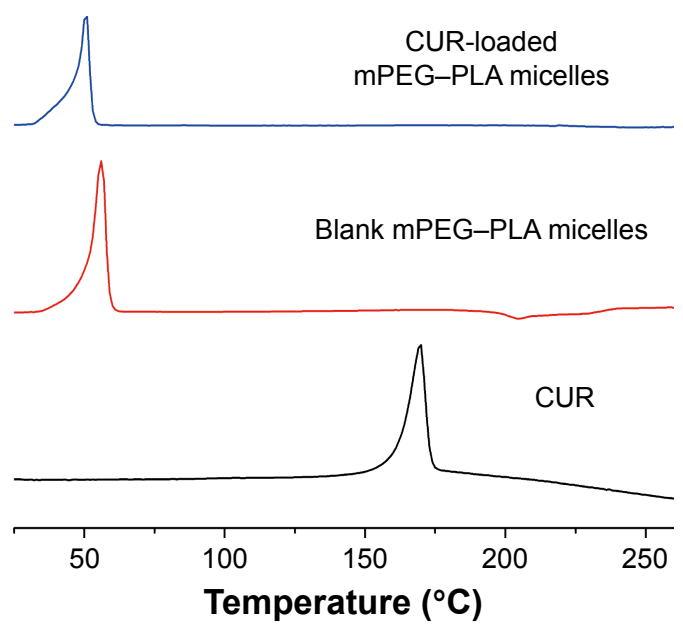

Figure 2 Confirmation of the absence of CUR crystals in the CUR/mPEG-PLA micelles.

Notes: (A) XRD analysis of CUR, physical mixture of CUR and mPEG-PLA, blank mPEG-PLA micelles and CUR/mPEG-PLA micelles. (B) DSC analysis of CUR, blank mPEG-PLA micelles and CUR/mPEG-PLA micelles.

Abbreviations: CUR, curcumin; DSC, differential scanning calorimetry; mPEG-PLA, methoxy polyethylene glycol-polylactide; XRD, X-ray diffraction.

the XRD diagrams of CUR, blank mPEG-PLA micelles and the physical mixture of CUR and the mPEG-PLA copolymer; the absence of specific diffraction peaks in the XRD spectra of CUR/mPEG-PLA micelles indicated that there are no CUR crystals in the CUR/mPEG-PLA micelles. The thermograms of CUR and CUR/mPEG-PLA micelles shown in Figure 2B also confirmed that CUR exhibited an endothermic melting peak at $171.3^{\circ} \mathrm{C}$; upon encapsulation inside the micelles, the melting peak of CUR disappeared. Thus, Figure 2 suggested that CUR was completely and amorphously encapsulated within the core-shell structure of particles.

\section{Cytotoxicity and cellular uptake of A549 cells}

The MTT assay was applied to investigate the cytotoxicity of A549 cells. As shown in Figure 3A, CUR/mPEG-PLA micelles had higher cytotoxicity against A549 cells compared with free CUR. Half maximal inhibitory concentration $\left(\mathrm{IC}_{50}\right)$ of CUR $/ \mathrm{mPEG}-\mathrm{PLA}$ micelles at 48 hours $(30.18 \mu \mathrm{g} / \mathrm{mL})$ was lower than that of free CUR $(36.69 \mu \mathrm{g} / \mathrm{mL})$. Meanwhile, cytotoxicity of mPEG-PLA copolymer was investigated on A549 cells (data not shown), suggesting that mPEG-PLA copolymer had low cytotoxicity and could serve as a safe carrier.

To understand why CUR/mPEG-PLA micelles may enhance cytotoxicity, cellular uptake ability was compared between CUR/mPEG-PLA micelles and free CUR on A549 cells. While there was no fluorescence in cells treated with blank mPEG-PLA micelles (data not shown), only a slight increase in fluorescence intensity was observed in cells treated with free CUR $(20 \mu \mathrm{g} / \mathrm{mL})$ for either 1 or 4 hours. On the contrary, bright green fluorescence was observed for CUR/ mPEG-PLA micelles $(20 \mu \mathrm{g} / \mathrm{mL})$ for 1 hour and even brighter for 4 hours (Figure 3B), clearly indicating that CUR/mPEGPLA micelles exhibited a much higher cellular uptake.

The enhanced cellular uptake of CUR/mPEG-PLA micelles was also confirmed by FCM analysis. Figure 3C-E shows that fluorescence intensity of cells treated with CUR/ mPEG-PLA micelles is much higher than that in free CUR group after incubation for either 1 or 4 hours $(P<0.01)$.

\section{CUR/mPEG-PLA micelles induce cell cycle arrest in A549 cells}

To evaluate whether CUR/mPEG-PLA micelles modulate cycle kinetics in A549 cells, cell cycle analysis was performed on A549 cells using FCM analysis after 24 hours of drug exposure. As shown in Figure 4A and C, the blank mPEGPLA micelles treatment showed no obvious effects on cell cycle distribution. However, at $20 \mu \mathrm{g} / \mathrm{mL}$, free CUR induced G2/M cell cycle arrest, resulting in a considerable increase in the $\mathrm{G} 2 / \mathrm{M}$ phase from $6.59 \% \pm 0.75 \%$ to $12.76 \% \pm 1.03 \%$ $(P<0.01)$. Furthermore, CUR/mPEG-PLA micelles gave rise to an even more obvious cell proliferation inhibition, where cell cycle was apparently retarded at the $\mathrm{G} 2 / \mathrm{M}$ transition point from $6.59 \% \pm 0.75 \%$ to $18.52 \% \pm 1.21 \%(P<0.01)$.

Cell colony formation unit assay was also carried out. As shown in Figure 4B and D, when A549 cells were treated with free CUR or CUR/mPEG-PLA micelles, the ability to form colonies was obviously changed compared to the untreated group. In addition, CUR/mPEG-PLA micelles exhibited less A549 cell numbers than free CUR groups $(P<0.01)$, 


\section{A}
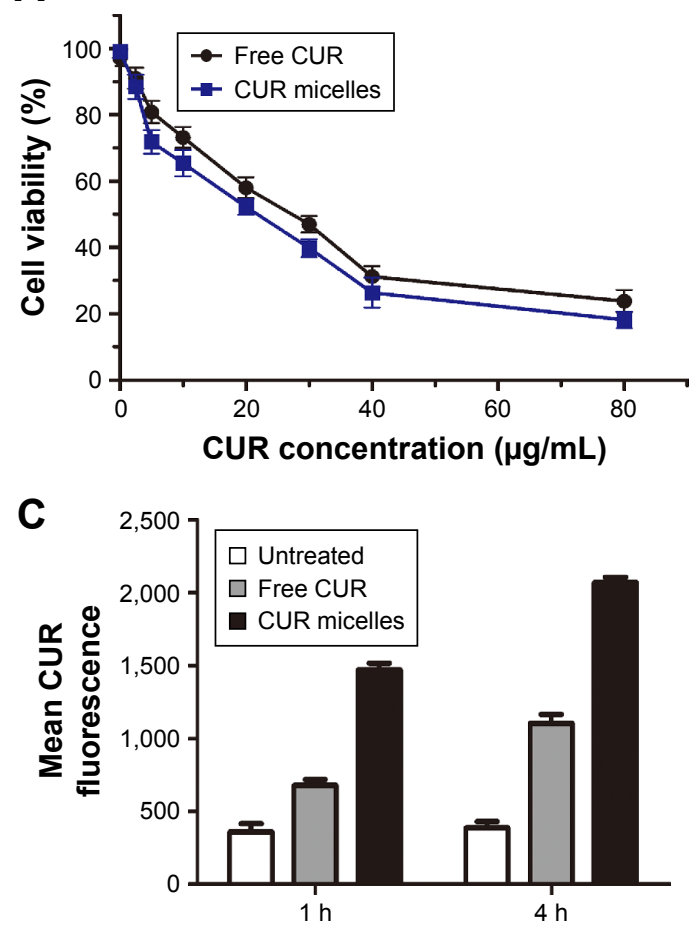

B
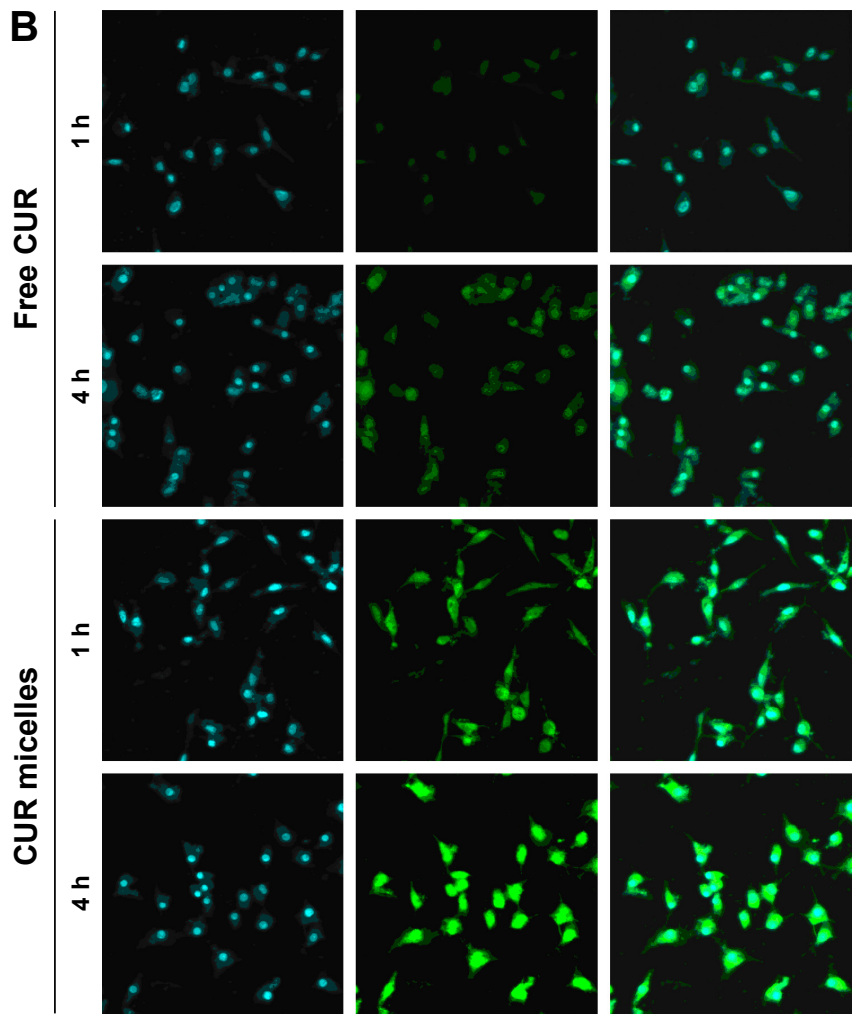

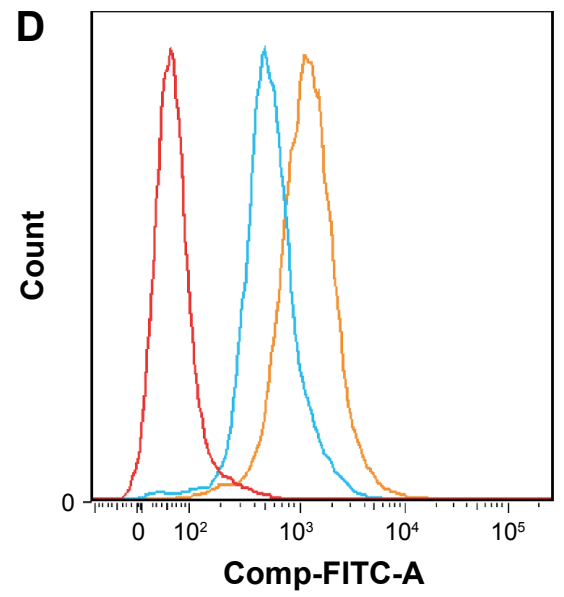

E

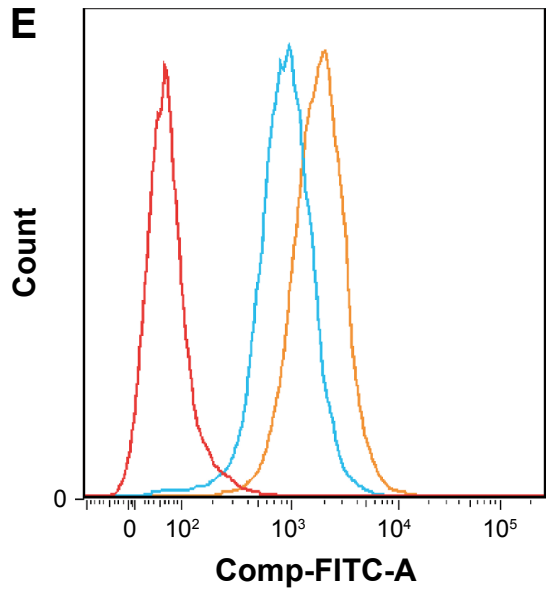

\section{Sample name}

Untreated

Free CUR

CUR micelles

Figure 3 Cytotoxicity studies and cellular uptake assay of CUR micelles.

Notes: (A) Cytotoxicity studies of A549 cells treated with free CUR and CUR micelles. (B) Fluorescent images of A549 cells treated with free CUR and CUR micelles under a confocal microscope at the indicated time intervals. Nuclei were stained blue with Hoechst 33258, and cellular distribution of CUR is shown as green fluorescence in the cytosol. (Magnifcation 400×) (C) CUR fluorescence means accumulation in A549 cells. (D) FCM histogram for free CUR and CUR micelles at $20 \mu \mathrm{g} / \mathrm{mL}$ on A549 cells for I hour. (E) FCM histogram for free CUR and CUR micelles at $20 \mu \mathrm{g} / \mathrm{mL}$ on A549 cells for 4 hours. CUR micelles, CUR/mPEG-PLA micelles.

Abbreviations: CUR, curcumin; FCM, flow cytometry; mPEG-PLA, methoxy polyethylene glycol-polylactide; h, hour.

indicating that CUR/mPEG-PLA micelles could inhibit the proliferation and clone formation ability of A549 cells.

\section{CUR/mPEG-PLA micelles induce apoptosis}

Cell apoptotic rate was analyzed via FCM analysis. As shown in Figure 5A, no apparent sign of cell apoptosis was observed in untreated cells or blank mPEG-PLA micelle-treated cells. However, treatment of CUR increased the apoptotic cell proportion from $5.96 \% \pm 0.18 \%$ to $16.33 \% \pm 0.68 \%(P<0.01)$. Furthermore, $\mathrm{CUR} / \mathrm{mPEG}-$ PLA micelles treatment increased the cell apoptotic rate from $5.96 \% \pm 0.18 \%$ to $24.93 \% \pm 0.51 \%$, especially at early stage (Figure 5B) $(P<0.01)$. These results displayed that 


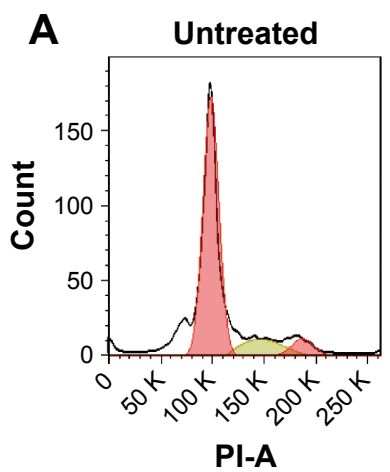

B

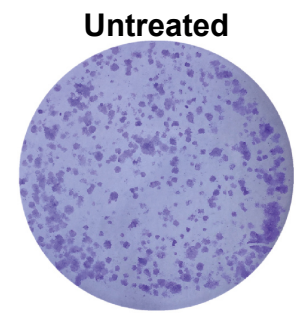

C

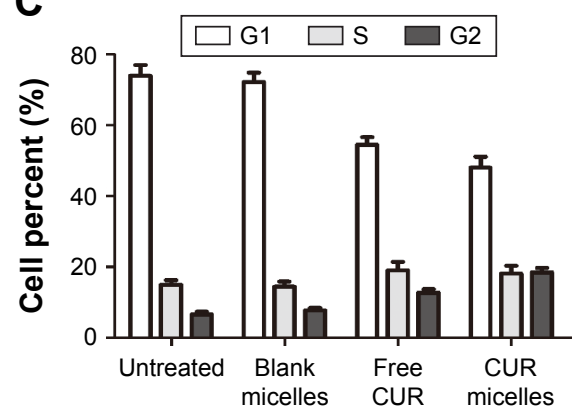

Blank micelles

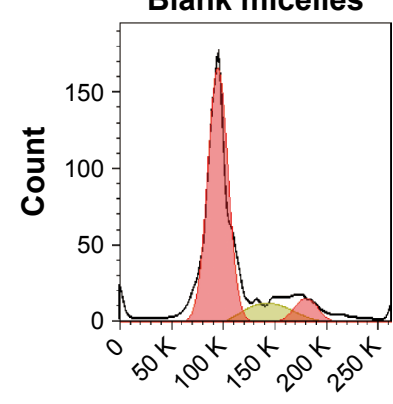

PI-A
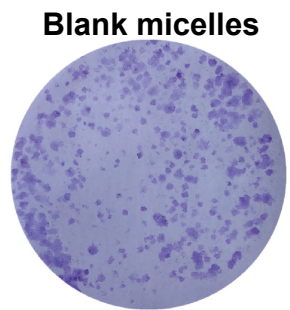

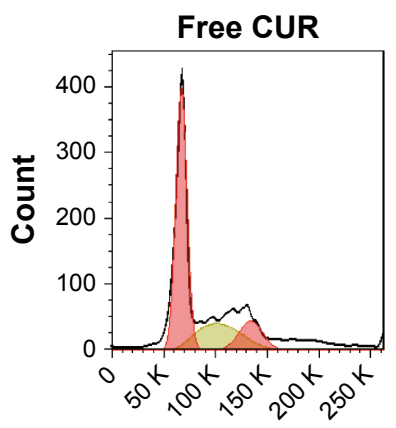

PI-A

Free CUR

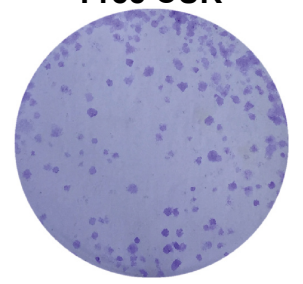

D

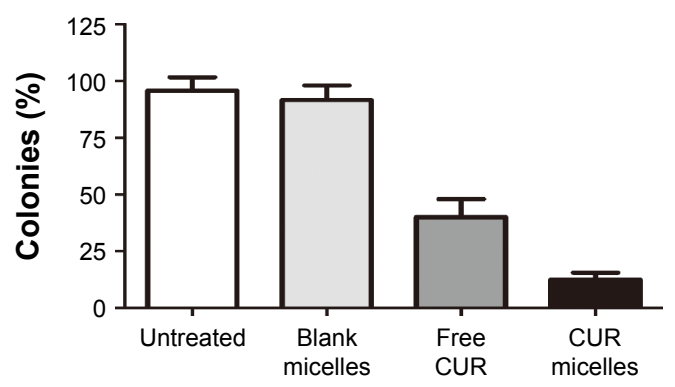

Figure 4 CUR micelles induce cell cycle arrest in A549 cells.

Notes: (A) Cell cycle distribution of A549 cells treated with free CUR and CUR micelles. (B) Clonogenic survival assay of A549 cells treated with free CUR or CUR micelles. (C) The histograms exhibit percent of cells in different phages of cell cycle. (D) Analysis of colonies per dish, represented as a percent of control. Blank micelles, blank mPEG-PLA micelles; CUR micelles, CUR/mPEG-PLA micelles.

Abbreviations: CUR, curcumin; mPEG-PLA, methoxy polyethylene glycol-polylactide.

CUR/mPEG-PLA micelles induced intense apoptosis in A549 cells.

Apoptosis is a course of a series of gene activation and regulation. The protein level of $\mathrm{Bax} / \mathrm{Bcl}-2$ was increased in CUR/mPEG-PLA micelles group compared with free CUR group (Figure 5C), suggesting an increased apoptosis by CUR/mPEG-PLA micelles in comparison with free CUR. In addition, the caspase 3 kit analysis showed that CUR/mPEG-PLA micelles induced more caspase 3 activation than free CUR (Figure 5D) $(P<0.01)$, further supporting the enhanced proapoptotic effect of CUR/mPEGPLA micelles.

\section{CUR/mPEG-PLA micelles inhibit the metastasis of lung cancer cells}

Treatment with free CUR and CUR/mPEG-PLA micelles significantly reduced A549 cell migration compared to untreated cells $(P<0.01)$ (Figure 6A and $\mathrm{C})$. Moreover, the inhibitory migration effect of CUR/mPEG-PLA micelles was significantly higher than that of free CUR $(P<0.01)$. CUR/mPEG-PLA micelles also showed a stronger inhibitory effect than free CUR in the transwell invasion assay $(P<0.01)$ (Figure 6B and D). These findings clearly revealed that CUR inhibited the migration and invasion on lung cancer cells. Moreover, this activity was related to the decreased expression of VEGF, MMP-2 and MMP-9 in human lung cancer A549 cells (Figure 6E).

\section{In vitro antiangiogenic activity of CUR/mPEG-PLA micelles}

Angiogenesis is a very complex process. HUVECs are usually used to evaluate the anti-angiogenesis activity in vitro. ${ }^{24}$ As shown in Figure 7A and E, HUVECs formed capillary-like structures on the surface of Matrigel within 


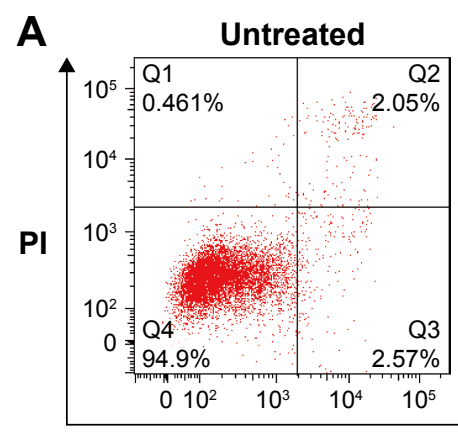

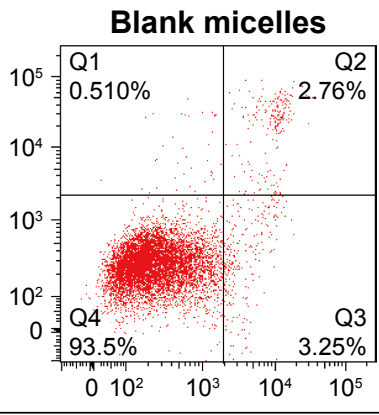
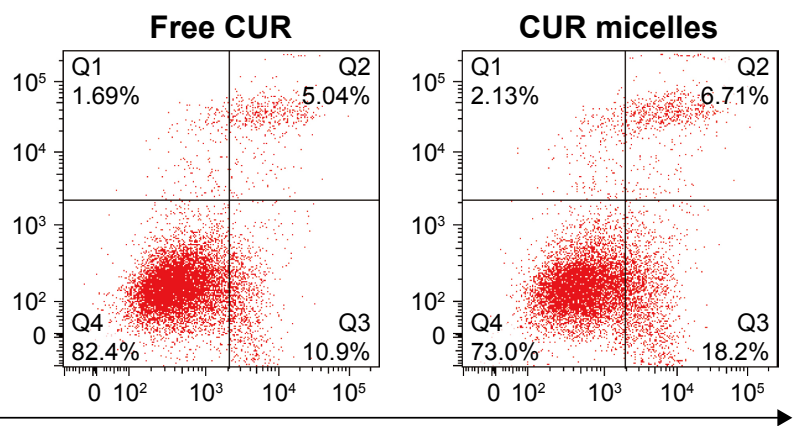

Annexin V-FITC

B

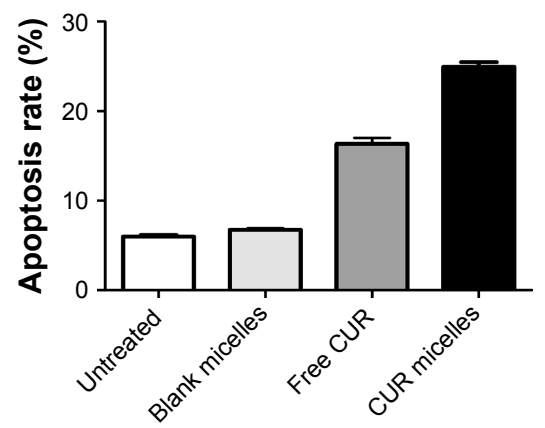

C

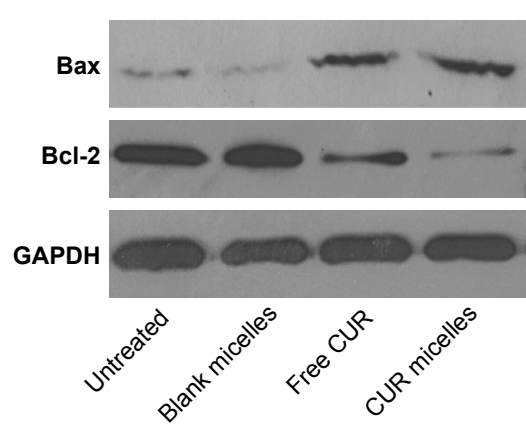

D

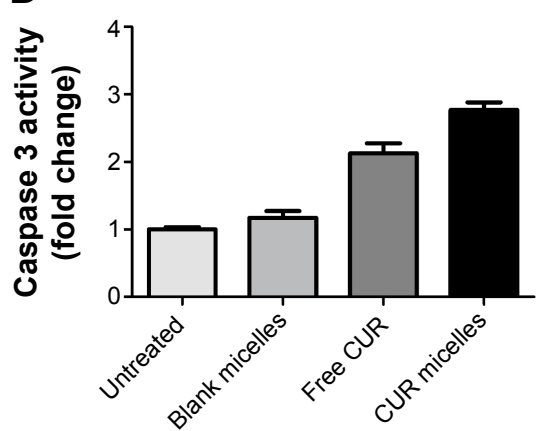

Figure 5 CUR micelles induced A549 cells apoptosis and caspase 3 activation.

Notes: (A) The apoptosis incidence of A549 cells was evaluated by FCM; cells were treated with blank micelles, free CUR and CUR micelles, respectively. (B) Statistical analysis of apoptotic rate was calculated via the percentage of apoptotic cells. (C) Western blotting for the expression of proteins related to apoptosis Bax and Bcl-2, GAPDH acted as a loading control. (D) Changes of Caspase 3 activity. Blank micelles, blank mPEG-PLA micelles; CUR micelles, CUR/mPEG-PLA micelles.

Abbreviations: CUR, curcumin; FCM, flow cytometry; GAPDH, glyceraldehyde 3-phosphate dehydrogenase; mPEG-PLA, methoxy polyethylene glycol-polylactide.

$6 \mathrm{~h}$ in untreated and blank mPEG-PLA micelles group. In contrast, the tube formation of HUVECs was inhibited by treating with free CUR or CUR/mPEG-PLA micelles, and CUR/mPEG-PLA micelles induced a stronger inhibitory effect than free CUR $(P<0.01)$. The effects of the CUR/ $\mathrm{mPEG}-\mathrm{PLA}$ micelles on the migration and invasion capacity of HUVECs were evaluated by wound-healing assay and transwell invasion assay, respectively. It was shown that the migration ability of HUVECs was inhibited in both CUR/ mPEG-PLA micelles and free CUR groups (Figure 7B and F) and that CUR/mPEG-PLA micelles group exhibited a stronger inhibitory effect than free CUR group $(P<0.01)$. Transwell invasion assays were performed to evaluate the ability of HUVECs passing through the Matrigel in the presence of blank mPEG-PLA micelles, free CUR or CUR/mPEG-PLA micelles. As shown in Figure 7C and G, in comparison with free CUR group, the inhibitory effect was improved in the CUR/mPEG-PLA micelles group $(P<0.01)$. The MTT assay was performed to evaluate the cytotoxicity effects of CUR/ mPEG-PLA micelles on HUVECs. Figure 7D showed that the growth ability of HUVECs was dramatically inhibited in a dose-dependent manner in both free CUR and CUR/ mPEG-PLA micelles groups and that CUR/mPEG-PLA micelles had a lower $\mathrm{IC}_{50}(31.39 \mu \mathrm{g} / \mathrm{mL})$ than free CUR (43.02 $\mu \mathrm{g} / \mathrm{mL})$.

\section{Discussion}

As a popular natural medicine, CUR has been widely used for treating patients with a variety of tumors. ${ }^{25}$ However, its clinical application was restricted by its hydrophobicity. As such, increasing solubility of CUR is an urgent strategy to facilitate its clinical application. ${ }^{26,27}$ In this study, CUR/mPEG-PLA micelles were successfully developed, and their antitumor and anti-angiogenesis activity suggested their clinical potential for treating NSCLC.

Nanometer material has been reported to successfully enhance solubility and biological availability of hydrophobic drugs. ${ }^{28-30}$ Previously, mPEG-PLA has been employed as nanometer material to deliver doxorubicin, ${ }^{31}$ paclitaxel $^{32}$ and 7-ethyl-10-hydroxy camptothecin ${ }^{33}$ for cancer therapy, leading to a considerable improvement in their anticancer effects. As an amphiphilic block copolymer composed of hydrophilic and hydrophobic segments, mPEG-PLA has a tendency to self-assemble into micelles in a specific solvent. In this study, a thin-film hydration method was applied to obtain CUR/mPEG-PLA micelles. Such CUR/mPEG-PLA 


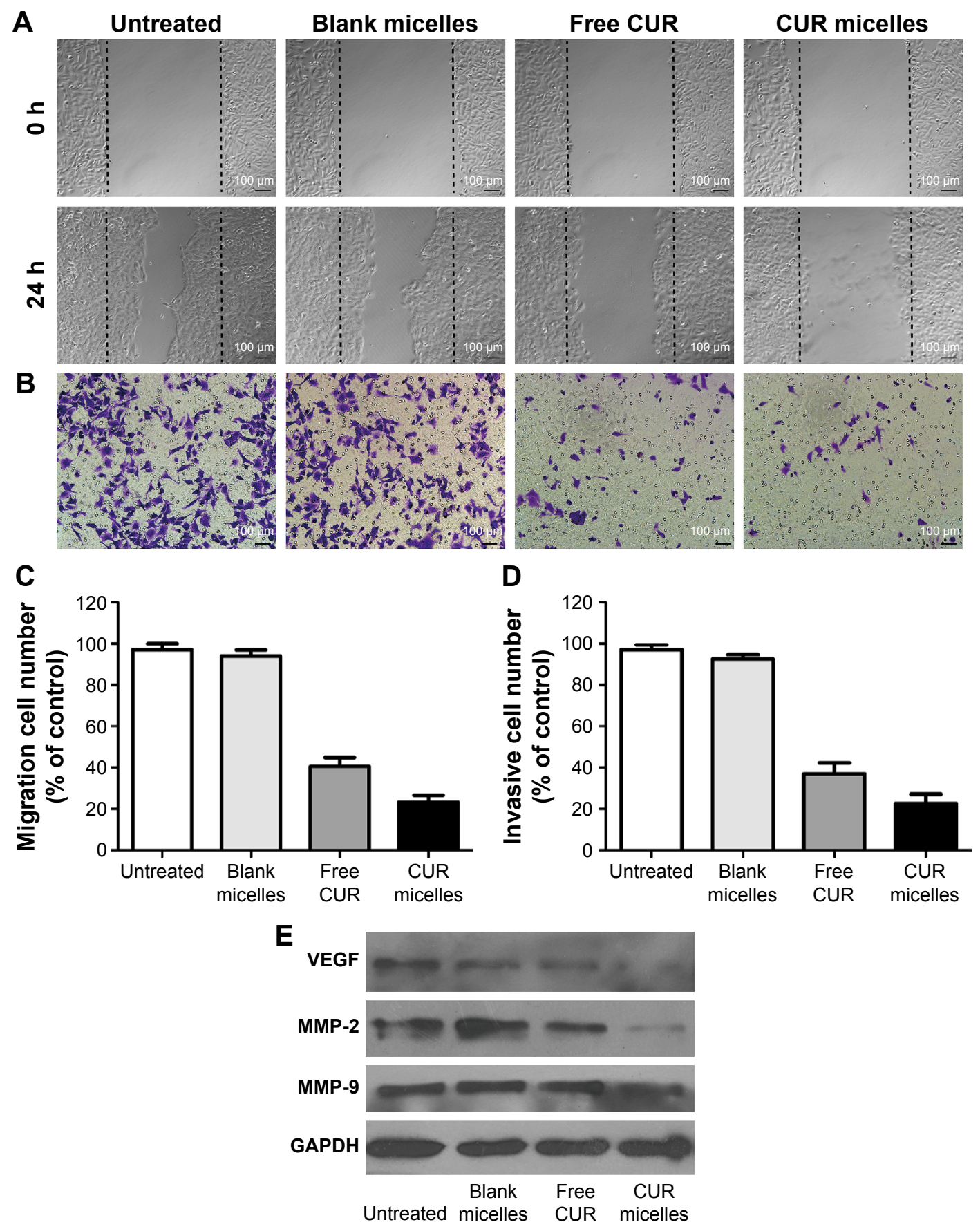

Figure 6 CUR micelles inhibited the migration and invasion of A549 cells in vitro.

Notes: (A and C) CUR micelles inhibited A549 cell migration in wound-healing assay. (B and D) CUR micelles inhibited A549 cell invasion in transwell assay. Images in (A and B) were taken by microscope under $\times 100$ magnification. (E) The VEGF, MMP-2 and MMP-9 were analyzed by Western blotting assay, with GAPDH acted as loading controls. Blank micelles, blank mPEG-PLA micelles; CUR micelles, CUR/mPEG-PLA micelles.

Abbreviations: CUR, curcumin; GAPDH, glyceraldehyde 3-phosphate dehydrogenase; MMP, matrix metalloproteinase; mPEG-PLA, methoxy polyethylene glycolpolylactide; VEGF, vascular endothelial growth factor.

micelles were monodispersed with PDI of $0.1 \pm 0.04$ in aqueous phase. Meanwhile, the CUR/mPEG-PLA micelles were characterized with stable DL, high EE and slow release behavior, which may be attributed to the core-shell structure of polymeric micelles. ${ }^{34}$ It has been confirmed that drug-loaded micelles with small size $(10-100 \mathrm{~nm})$ could increase the accumulation in tumor tissue by enhancing permeability and retention (EPR) effect, ${ }^{35}$ therefore improving antitumor effects. The CUR/mPEG-PLA micelles prepared in this study have an average diameter of 

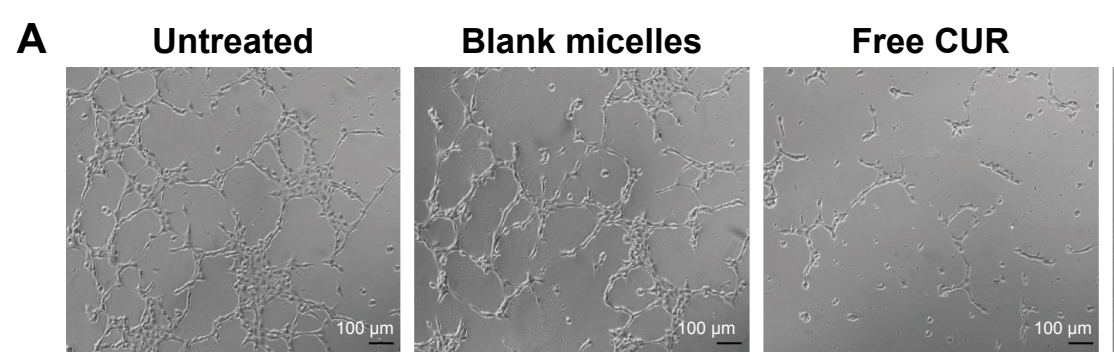

\section{CUR micelles}
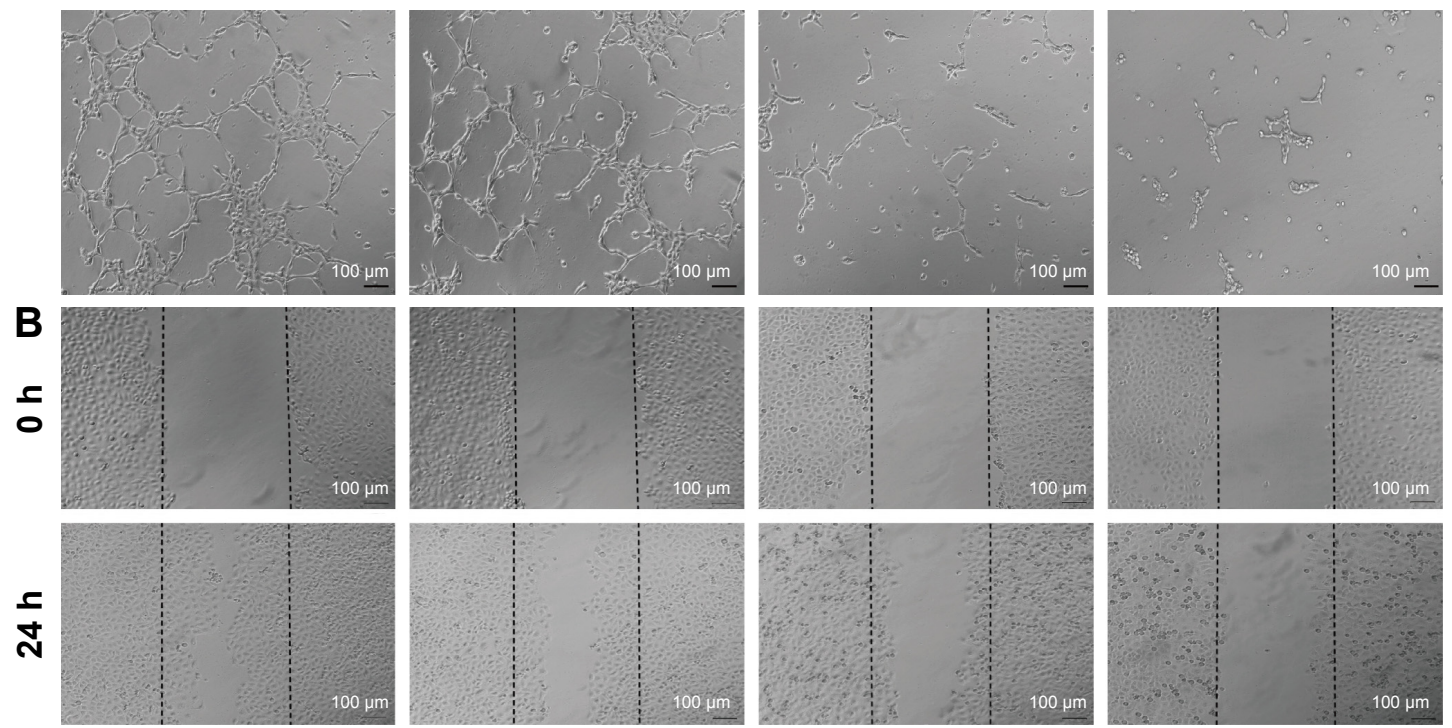

C
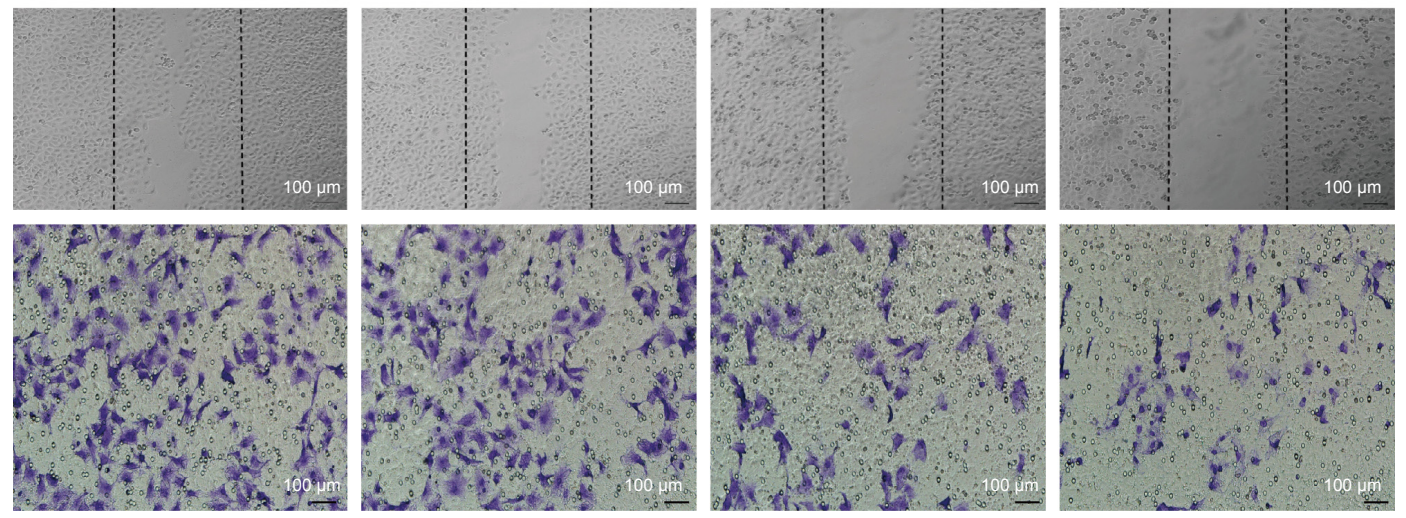

D
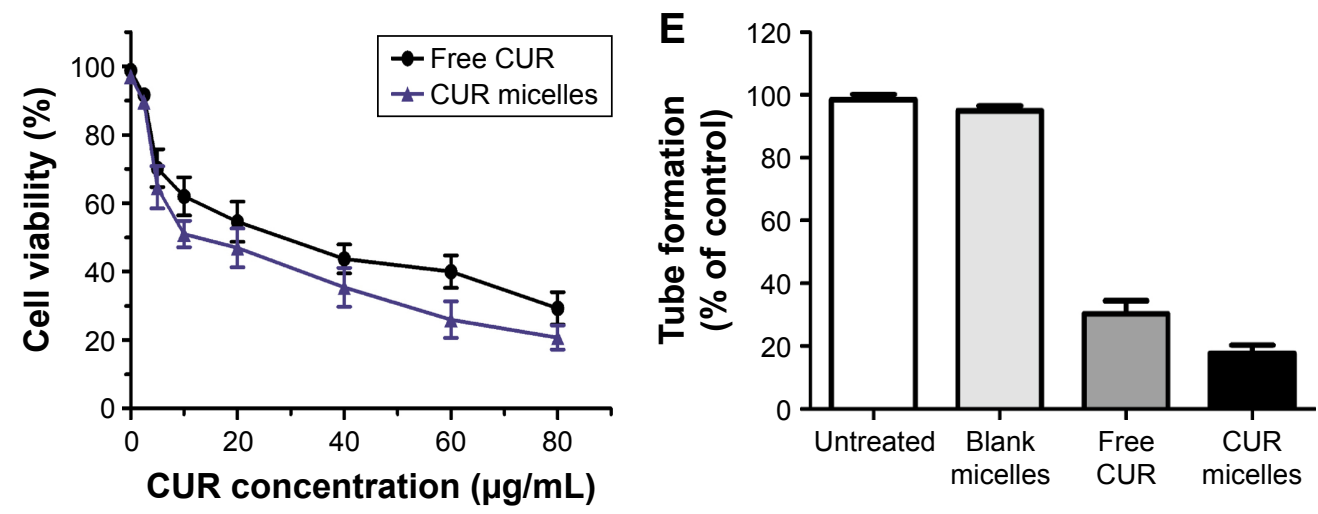

$\mathbf{F}$
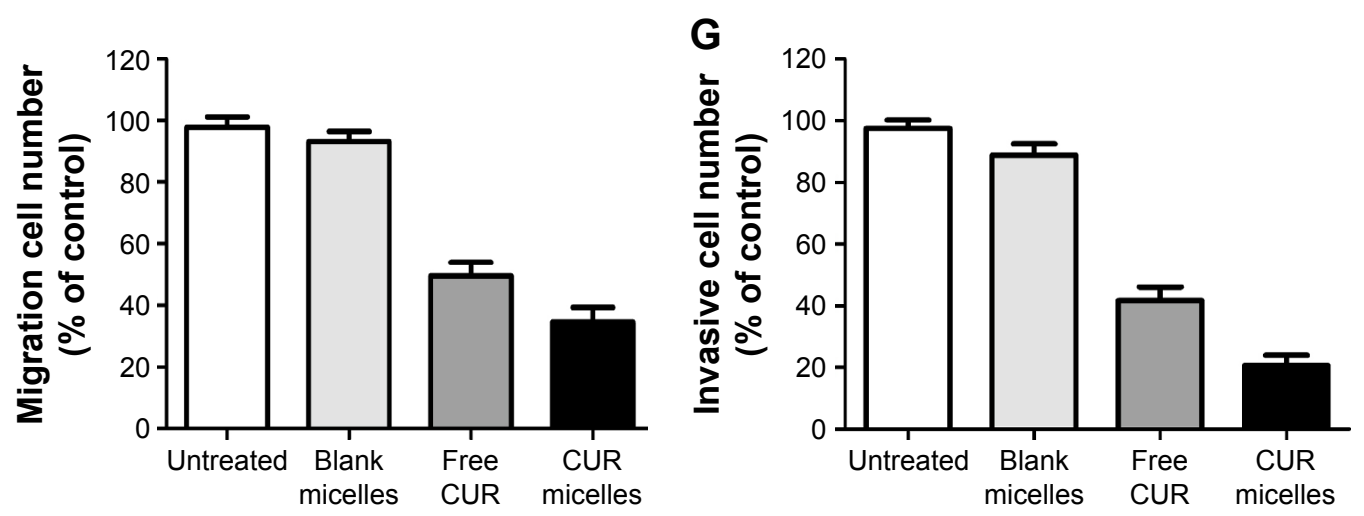

Figure 7 CUR micelles induced cytotoxicity and inhibited tube formation, migration and invasion of HUVECs in vitro.

Notes: (A and E) CUR micelles inhibited tube formation of HUVECs. (B and F) CUR micelles inhibited HUVECs migration in wound-healing assay. (C and G) CUR micelles inhibited HUVECs invasion in transwell assay. Images in (A-C) were taken by microscope under $\times 100$ magnification. (D) CUR micelles enhanced the cytotoxicity of CUR of HUVECs. Blank micelles, blank mPEG-PLA micelles; CUR micelles, CUR/mPEG-PLA micelles.

Abbreviations: CUR, curcumin; HUVECs, human umbilical vein endothelial cells; mPEG-PLA, methoxy polyethylene glycol-polylactide. 
$34.9 \pm 2.1 \mathrm{~nm}$, which, together with its considerably enhanced cellular uptake, should contribute to its good antitumor potentials. A series of experiments was performed in this study to evaluate the antitumor effect of CUR/mPEG-PLA micelles in A549 cells. Our results showed that CUR/mPEG-PLA micelles enhanced cytotoxicity and decreased $\mathrm{IC}_{50}$ cell viability, whereas mPEG-PLA exhibited low cytotoxicity to A549 cells. It was previously reported that both free CUR and CUR nanomaterials were nontoxic to human bronchus epithelial cell line BEAS-2B (normal cell). ${ }^{36,37}$ As such, CUR/mPEGPLA micelles are considered to be safe to normal cells, but to be lethal to tumor cells. The CUR/mPEG-PLA micelles decreased the colony formation unit number as compared with free CUR group. It was also found that the proportion of cells in the G2/M was increased in CUR/mPEG-PLA micelles group. These findings indicated that CUR/mPEGPLA micelle-induced cell growth inhibition was involved with cell cycle arrest. Apoptosis plays an important role in the initiation and progression of tumor, and $\mathrm{Bax}$ and $\mathrm{Bcl}-2$ family pathway is involved in regulating cell propagation and cell apoptosis. ${ }^{38}$ Our results showed that CUR/mPEG-PLA micelles may enhance A549 cells apoptosis and significantly increased the expression of Bax and reduced the expression of Bcl-2. Moreover, CUR/mPEG-PLA micelles increased caspase 3 activity, which is the final stage of apoptosis initiation shared by both pathways. ${ }^{39}$

Metastasis is a complex multistep process that requires detachment from the primary tumor mass, migration through the extracellular matrix (ECM) and the colonization of surrounding sites. ${ }^{40}$ The migration assay and invasion assay demonstrated that CUR/mPEG-PLA micelles significantly inhibited A549 cells migration and invasion than free CUR. One of the key factors associated with the invasion and metastasis of cancer cells is MMPs, which led cancer cells to invade and migrate by degrading the ECM. Among MMPs, MMP-2 and MMP-9 expression is correlated with lung cancer metastasis. ${ }^{41,42}$ Additionally, VEGF, the key mediator of angiogenesis, also plays a crucial role in the process of metastasis. $^{43}$ Therefore, the protein levels of MMP-2, MMP-9 and VEGF in A549 cells were evaluated. Our results that much lower expression of the abovementioned proteins in CUR/mPEG-PLA micelles group than in the free CUR group clearly indicated that CUR/mPEG-PLA micelles may induce apoptosis and inhibit cell growth, migration and invasion.

Angiogenesis plays a vital role in tumor growth and metastasis via supplying oxygen and nutrient. ${ }^{44,45}$ Antiangiogenesis has been proposed as a promising therapeutic strategy for clinical therapy of tumors, ${ }^{46,47}$ mainly by slowing down tumor dissemination and increasing tumor responses to chemotherapeutics and radiotherapy. ${ }^{48}$ Clinical trial data show that several drugs, including bevacizumab, play an important role in the treatment of advanced NSCLC by targeting VEGF pathway. ${ }^{49}$ Therefore, the effect of CUR micelles was further investigated on angiogenesis in HUVECs in vitro. Our results showed that CUR/mPEG-PLA micelles induced cytotoxicity to HUVECs and inhibited the migration, invasion and tube formation of HUVECs, which was consistent with their effect on colon cancer. ${ }^{50}$ It is therefore believed that CUR/mPEG-PLA micelles can effectively inhibit the angiogenesis.

\section{Conclusion}

CUR/mPEG-PLA micelles were successfully prepared by a thin-film hydration method, with a high EE, and sustained release behavior in vitro. It was suggested that CUR/mPEGPLA micelles are capable of enhancing the cellular uptake, cytotoxicity and apoptosis, while inhibiting the proliferation, migration and metastasis of A549 cells. Their angiogenesis ability was also confirmed in HUVECs. Our findings suggested that this novel formulation has potential clinical application for treating NSCLC. However, further in vivo investigations are warranted using A549 cell-bearing tumor model to better understand their antitumor potential.

\section{Acknowledgments}

This study was supported by the National Natural Science Foundations of China (Nos 81373365 and 81572219) and Guangdong Science and Technology Project (No 2015A010105014).

\section{Disclosure}

The authors report no conflicts of interest in this work.

\section{References}

1. Torre LA, Bray F, Siegel RL, Ferlay J, Lortet-Tieulent J, Jemal A. Global cancer statistics, 2012. CA Cancer J Clin. 2015;65(2):87-108.

2. Hirsch FR, Suda K, Wiens J, Bunn PA Jr. New and emerging targeted treatments in advanced non-small-cell lung cancer. Lancet. 2016; 388(10048): 1012-1024.

3. Pilkington G, Boland A, Brown T, Oyee J, Bagust A, Dickson R. A systematic review of the clinical effectiveness of first-line chemotherapy for adult patients with locally advanced or metastatic non-small cell lung cancer. Thorax. 2015;70(4):359-367.

4. Ye MX, Li Y, Yin H, Zhang J. Curcumin: updated molecular mechanisms and intervention targets in human lung cancer. Int J Mol Sci. 2012; 13(3):3959-3978.

5. Duvoix A, Blasius R, Delhalle $\mathrm{S}$, et al. Chemopreventive and therapeutic effects of curcumin. Cancer Lett. 2005;223(2):181-190.

6. Devassy JG, Nwachukwu ID, Jones PJ. Curcumin and cancer: barriers to obtaining a health claim. Nutr Rev. 2015;73(3):155-165. 
7. Kumar G, Mittal S, Sak K, Tuli HS. Molecular mechanisms underlying chemopreventive potential of curcumin: current challenges and future perspectives. Life Sci. 2016;148:313-328.

8. Kasi PD, Tamilselvam R, Skalicka-Wozniak K, et al. Molecular targets of curcumin for cancer therapy: an updated review. Tumour Biol. 2016;37(10):13017-13028.

9. Ghosh S, Banerjee S, Sil PC. The beneficial role of curcumin on inflammation, diabetes and neurodegenerative disease: a recent update. Food Chem Toxicol. 2015;83:111-124.

10. Anand P, Kunnumakkara AB, Newman RA, Aggarwal BB. Bioavailability of curcumin: problems and promises. Mol Pharm. 2007;4(6): 807-818.

11. Wang S, Su R, Nie S, et al. Application of nanotechnology in improving bioavailability and bioactivity of diet-derived phytochemicals. J Nutr Biochem. 2014;25(4):363-376.

12. Jeetah R, Bhaw-Luximon A, Jhurry D. Nanopharmaceutics: phytochemical-based controlled or sustained drug-delivery systems for cancer treatment. J Biomed Nanotechnol. 2014;10(9):1810-1840.

13. Gharpure KM, Wu SY, Li C, Lopez-Berestein G, Sood AK. Nanotechnology: future of oncotherapy. Clin Cancer Res. 2015;21(14):3121-3130.

14. Naksuriya O, Okonogi S, Schiffelers RM, Hennink WE. Curcumin nanoformulations: a review of pharmaceutical properties and preclinical studies and clinical data related to cancer treatment. Biomaterials. 2014;35(10):3365-3383.

15. Riela S, Massaro M, Colletti CG, et al. Development and characterization of co-loaded curcumin/triazole-halloysite systems and evaluation of their potential anticancer activity. Int J Pharm. 2014;475(1-2):613-623.

16. Yallapu MM, Jaggi M, Chauhan SC. Curcumin nanoformulations: a future nanomedicine for cancer. Drug Discov Today. 2012;17(1-2): 71-80.

17. Liu M, Du H, Zhai G. The design of amphiphilic polymeric micelles of curcumin for cancer management. Curr Med Chem. 2015;22(38): 4398-4411.

18. Yu Y, Zhang X, Qiu L. The anti-tumor efficacy of curcumin when delivered by size/charge-changing multistage polymeric micelles based on amphiphilic poly(beta-amino ester) derivates. Biomaterials. 2014;35(10):3467-3479

19. Mehanny M, Hathout RM, Geneidi AS, Mansour S. Exploring the use of nanocarrier systems to deliver the magical molecule; curcumin and its derivatives. J Control Release. 2016;225:1-30.

20. Zhao C, Deng H, Xu J, et al. "Sheddable" PEG-lipid to balance the contradiction of PEGylation between long circulation and poor uptake. Nanoscale. 2016;8(20):10832-10842.

21. Zheng S, Gao X, Liu X, et al. Biodegradable micelles enhance the antiglioma activity of curcumin in vitro and in vivo. Int J Nanomedicine. 2016;11:2721-2736.

22. Kumari P, Swami MO, Nadipalli SK, et al. Curcumin delivery by poly(lactide)-based Co-polymeric micelles: an in vitro anticancer study. Pharm Res. 2016;33(4):826-841.

23. Gong $\mathrm{C}$, Deng $\mathrm{S}, \mathrm{Wu} \mathrm{Q}$, et al. Improving antiangiogenesis and antitumor activity of curcumin by biodegradable polymeric micelles. Biomaterials. 2013;34(4):1413-1432.

24. Park HJ, Zhang Y, Georgescu SP, Johnson KL, Kong D, Galper JB. Human umbilical vein endothelial cells and human dermal microvascular endothelial cells offer new insights into the relationship between lipid metabolism and angiogenesis. Stem Cell Rev. 2006;2(2):93-102.

25. Shanmugam MK, Rane G, Kanchi MM, et al. The multifaceted role of curcumin in cancer prevention and treatment. Molecules. 2015; 20(2):2728-2769.

26. Tang H, Murphy CJ, Zhang B, et al. Curcumin polymers as anticancer conjugates. Biomaterials. 2010;31(27):7139-7149.

27. Dhillon N, Aggarwal BB, Newman RA, et al. Phase II trial of curcumin in patients with advanced pancreatic cancer. Clin Cancer Res. 2008; 14(14):4491-4499.
28. Mohanty C, Sahoo SK. The in vitro stability and in vivo pharmacokinetics of curcumin prepared as an aqueous nanoparticulate formulation. Biomaterials. 2010;31(25):6597-6611.

29. Mahmood K, Zia KM, Zuber M, Salman M, Anjum MN. Recent developments in curcumin and curcumin based polymeric materials for biomedical applications: a review. Int J Biol Macromol. 2015;81: 877-890.

30. Massaro M, Amorati R, Cavallaro G, et al. Direct chemical grafted curcumin on halloysite nanotubes as dual-responsive prodrug for pharmacological applications. Colloids Surf B Biointerfaces. 2016;140:505-513.

31. Jin X, Zou B, Luo L, et al. Codelivery of thioridazine and doxorubicin using nanoparticles for effective breast cancer therapy. Int $J$ Nanomedicine. 2016;11:4545-4552.

32. Yu Y, Qiu L. Optimizing particle size of docetaxel-loaded micelles for enhanced treatment of oral epidermoid carcinoma. Nanomedicine. 2016;12(7):1941-1949.

33. Xie J, Zhang X, Teng M, et al. Synthesis, characterization, and evaluation of mPEG-SN38 and mPEG-PLA-SN38 micelles for cancer therapy. Int J Nanomedicine. 2016;11:1677-1686.

34. Oerlemans C, Bult W, Bos M, Storm G, Nijsen JF, Hennink WE. Polymeric micelles in anticancer therapy: targeting, imaging and triggered release. Pharm Res. 2010;27(12):2569-2589.

35. Fang J, Nakamura $H$, Maeda $H$. The EPR effect: unique features of tumor blood vessels for drug delivery, factors involved, and limitations and augmentation of the effect. Adv Drug Deliv Rev. 2011;63(3):136-151.

36. Lee WH, Loo CY, Ong HX, Traini D, Young PM, Rohanizadeh R. Synthesis and characterization of inhalable flavonoid nanoparticle for lung cancer cell targeting. J Biomed Nanotechnol. 2016;12(2):371-386.

37. Chang HB, Chen BH. Inhibition of lung cancer cells A549 and H460 by curcuminoid extracts and nanoemulsions prepared from Curcuma longa Linnaeus. Int J Nanomedicine. 2015;10:5059-5080.

38. Zheng JH, Viacava Follis A, Kriwacki RW, Moldoveanu T. Discoveries and controversies in BCL-2 protein-mediated apoptosis. FEBS J. 2016;283(14):2690-2700.

39. Shalini S, Dorstyn L, Dawar S, Kumar S. Old, new and emerging functions of caspases. Cell Death Differ. 2015;22(4):526-539.

40. Hynes RO. Metastatic potential: generic predisposition of the primary tumor or rare, metastatic variants-or both? Cell. 2003;113(7):821-823.

41. Curran S, Murray GI. Matrix metalloproteinases: molecular aspects of their roles in tumour invasion and metastasis. Eur J Cancer. 2000; 36(13 Spec No):1621-1630.

42. Deryugina EI, Quigley JP. Tumor angiogenesis: MMP-mediated induction of intravasation- and metastasis-sustaining neovasculature. Matrix Biol. 2015;44-46:94-112.

43. McMahon G. VEGF receptor signaling in tumor angiogenesis. Oncologist. 2000;5(suppl 1):3-10.

44. Liotta LA, Steeg PS, Stetler-Stevenson WG. Cancer metastasis and angiogenesis: an imbalance of positive and negative regulation. Cell. 1991;64(2):327-336.

45. Hanahan D, Weinberg RA. Hallmarks of cancer: the next generation. Cell. 2011;144(5):646-674.

46. Jain RK. Normalization of tumor vasculature: an emerging concept in antiangiogenic therapy. Science. 2005;307(5706):58-62.

47. Aggarwal C, Somaiah N, Simon G. Antiangiogenic agents in the management of non-small cell lung cancer: where do we stand now and where are we headed? Cancer Biol Ther. 2012;13(5):247-263.

48. Carmeliet P, Jain RK. Molecular mechanisms and clinical applications of angiogenesis. Nature. 2011;473(7347):298-307.

49. Riely GJ, Miller VA. Vascular endothelial growth factor trap in non small cell lung cancer. Clin Cancer Res. 2007;13(15 pt 2):s4623-s4627.

50. Gou M, Men K, Shi H, et al. Curcumin-loaded biodegradable polymeric micelles for colon cancer therapy in vitro and in vivo. Nanoscale. 2011; 3(4):1558-1567. 


\section{Publish your work in this journal}

The International Journal of Nanomedicine is an international, peerreviewed journal focusing on the application of nanotechnology in diagnostics, therapeutics, and drug delivery systems throughout the biomedical field. This journal is indexed on PubMed Central, MedLine, CAS, SciSearch $\AA$, Current Contents $\AA /$ Clinical Medicine,

Journal Citation Reports/Science Edition, EMBase, Scopus and the Elsevier Bibliographic databases. The manuscript management system is completely online and includes a very quick and fair peer-review system, which is all easy to use. Visit http://www.dovepress.com/ testimonials.php to read real quotes from published authors.

Submit your manuscript here: http://www.dovepress.com/international-journal-of-nanomedicine-journal 